\title{
Atypical presentations of malignant otitis externa
}

\author{
Z Vourexakis, M-I Kos, J-P GuYot
}

\begin{abstract}
Introduction: Malignant otitis externa is a life-threatening infection of the skull base. Its presentation is not always typical.

Case reports: We report three cases of malignant otitis externa which illustrate the diversity of its clinical manifestations and the difficulties in its diagnosis.

Discussion: The perception of malignant otitis externa as an infection caused by Pseudomonas aeruginosa in diabetic patients is not always correct. The adoption of diagnostic criteria could be helpful in identifying atypical cases.
\end{abstract}

Key words: Otitis Externa; Malignant Otitis Externa; Necrotizing Otitis Externa; Pseudomonas

\section{Introduction}

In 1838, Toulmouche reported the case of a patient with progressive osteomyelitis of the temporal bone. This case is believed to represent the first report of malignant otitis externa. ${ }^{1}$ In 1959 , Meltzer and Keleman reported a case of external otitis due to Pseudomonas aeruginosa in a patient with poorly controlled diabetes mellitus, who later died of progressive osteomyelitis of the skull base, despite aggressive medical and surgical treatment. ${ }^{2}$ The term 'malignant otitis externa' was introduced by Chandler in 1968, in a report of 13 cases which attempted to describe the clinical and histological findings as well as the pathophysiological mechanisms of the disease. ${ }^{3}$ Later, the term 'necrotising otitis externa' was suggested in order to avoid confusion with true malignancies.

During the last four decades, many studies on malignant otitis externa have been published and much progress has been made in the diagnosis and treatment of the disease. Today, the initial 1970 s concept of potentially fatal external otitis caused by $P$ aeruginosa in patients with poorly controlled diabetes mellitus is considered to relate only to a subgroup of patients with malignant otitis externa.

The aim of the current study is to illustrate, by reporting three supposedly 'atypical' cases, the range of diversity in malignant otitis externa presentation, which may challenge clinical diagnosis.

\section{Case reports}

Case one

A 70-year-old, diabetic woman had originally presented complaining of right ear otorrhoea accompanied by severe headache. Initial physical examination had revealed otitis externa with associated perforation of the tympanic membrane. A computed tomography (CT) scan had revealed opacity of the right middle ear and mastoid, extensive inflammation of the periauricular and parapharyngeal spaces, and anterior displacement of the mandibular condyle. Staphylococcus aureus had been cultured from the external acoustic meatus. A diagnosis of malignant otitis externa had been made and antibiotic treatment had been initiated.

However, after two months' intravenous antibiotic treatment the patient's headaches and external acoustic meatus stenosis persisted, and she was referred from Tunisia to our hospital in Switzerland.

On admission to our department, clinical examination revealed oedema of the skin of the external auditory canal, with granulations, as well as purulent discharge from the pharyngeal opening of the eustachian tube.

A new CT scan showed progression of the inflammatory process, with evidence of skull base osteomyelitis (Figure 1) and an abscess in the nasopharynx and the temporomandibular joint.

We performed a mastoidectomy. There was diffuse inflammation of the mastoid mucosa, with no evident pus or lytic lesions. Histological analysis showed the presence of fungal hyphae, and cultures grew Aspergillus flavus.

A diagnosis of fungal malignant otitis externa was established. A two-week course of intravenous amphotericin B was commenced, followed by a six-week course of oral voriconazole. The clinical response was rapid and favourable, with disappearance of symptoms after two weeks of treatment.

One month after the initiation of antifungal treatment, a magnetic resonance imaging (MRI) scan showed only slight radiological improvement. As the patient continued to be practically free of symptoms, she was discharged from our hospital, returned to Tunisia and was lost to our follow up.

\section{Case two}

A 68-year-old man, in good general health, had consulted several doctors about persistent otalgia in his right ear. Otitis externa had been diagnosed and treated with local antibiotics and corticosteroids. However, after three months of this treatment the otalgia still persisted, and the patient's general condition started to deteriorate 


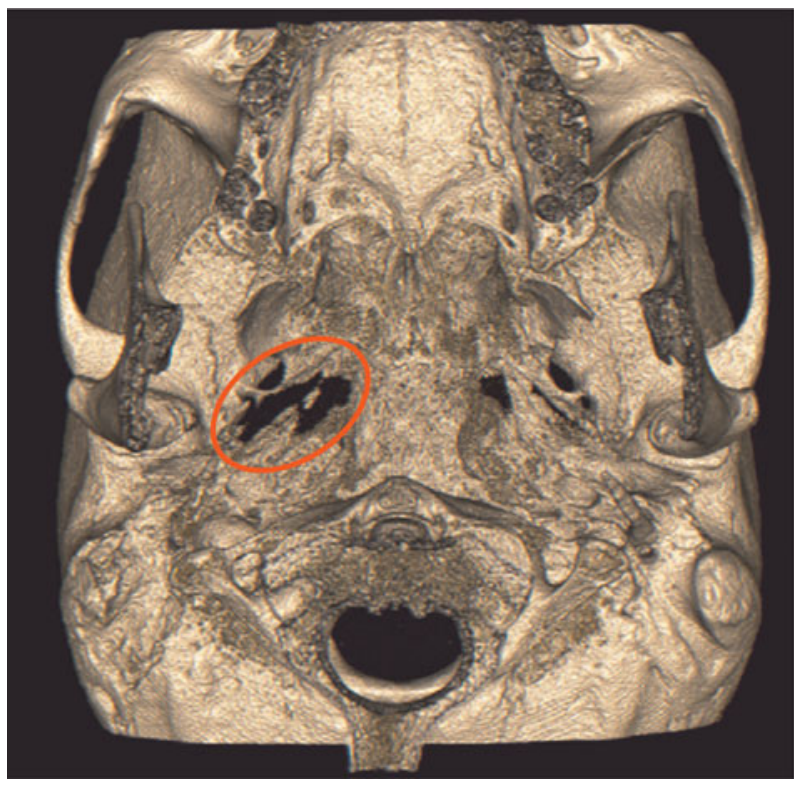

FIG. 1

Case one: reconstructed, three-dimensional computed tomography scan showing an extensive, osteolytic process of the skull base (oval).

rapidly, with severe headaches and a weight loss of $12 \mathrm{~kg}$ in two weeks.

At this time, ENT examination in our hospital revealed a right-ear otitis externa accompanied by oedema and erythema of the right nasopharyngeal walls. A CT-scan revealed an osteolytic process of the skull base with soft tissue thickening extending to the naso- and oro-pharynx, the prevertebral and parapharyngeal space (Figure 2).

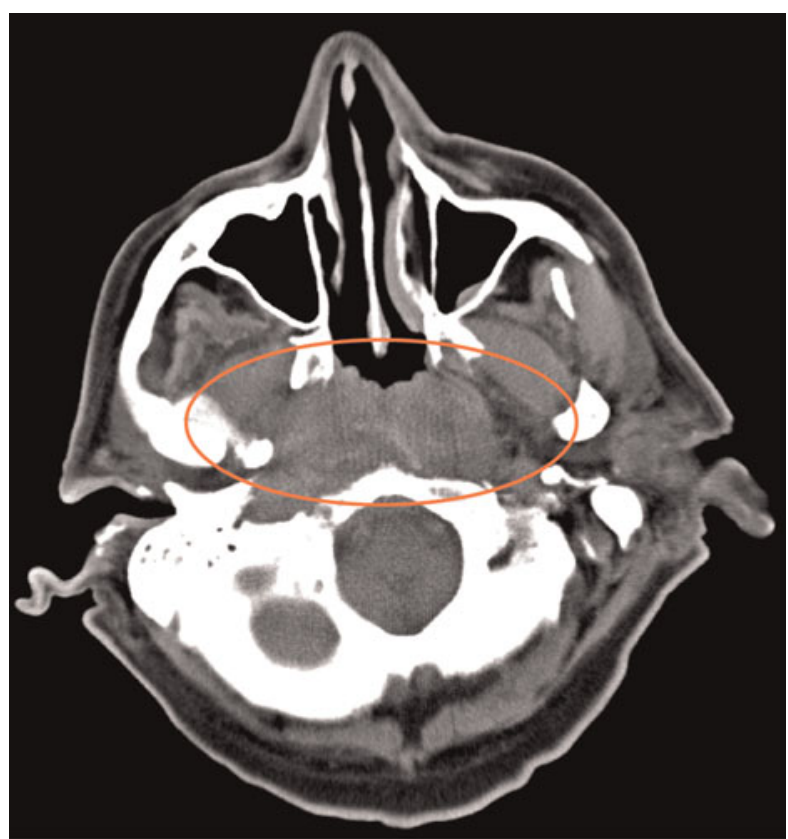

FIG. 2

Case two: axial computed tomography scan showing a significant but nonspecific thickening of the posterior pharyngeal wall (oval). Biopsies showed infiltration of the soft tissues by inflammatory cells.
Nasopharyngeal biopsies revealed a diffuse inflammatory cell infiltration, without malignancy. Capillary and venous blood glucose levels were normal.

Almost four months after his initial complaints, the patient required emergency admission, with vertigo and a third degree, left-beating, spontaneous nystagmus.

At mastoidectomy, mastoid and middle-ear tissue biopsies again showed diffuse inflammation, without malignant cells. However, tissue cultures showed a polymicrobial infection, predominantly with $P$ aeruginosa. A diagnosis of malignant otitis externa was suggested, and treatment with oral ciprofloxacin was initiated. The patient showed rapid recovery, with resolution of all symptoms.

In the long term, the patient experienced only a slight right ear sensorineural hearing loss, together with a positive head-thrust test to the right; however, he had no subjective complaints.

\section{Case three}

A 63-year-old man, with no history of diabetes, consulted an ENT specialist because of right otalgia. Otitis externa was diagnosed and topical treatment prescribed.

Five days later, the patient was admitted to the emergency department because of agitation and confusion. The otoscopic findings were still compatible with otitis externa. The ear drum was not visualised, and the postauricular region appeared to be normal. A CT scan revealed opacity of the right middle ear and mastoid, a thrombosis of the right transverse and sigmoid sinus (Figure 3), and a hypodense lesion of the right temporal lobe.

We performed an emergency mastoidectomy for debridement and drainage of a potential abscess. Surprisingly, despite significant inflammation of the mucosa, no pus was found in the mastoid. A polyp, surrounded by a small quantity of exudate, was identified in the medial part of the external acoustic meatus. Microbiological analysis revealed a polymicrobial infection with $P$ aeruginosa predominating. Intravenous antibiotic treatment was initiated.

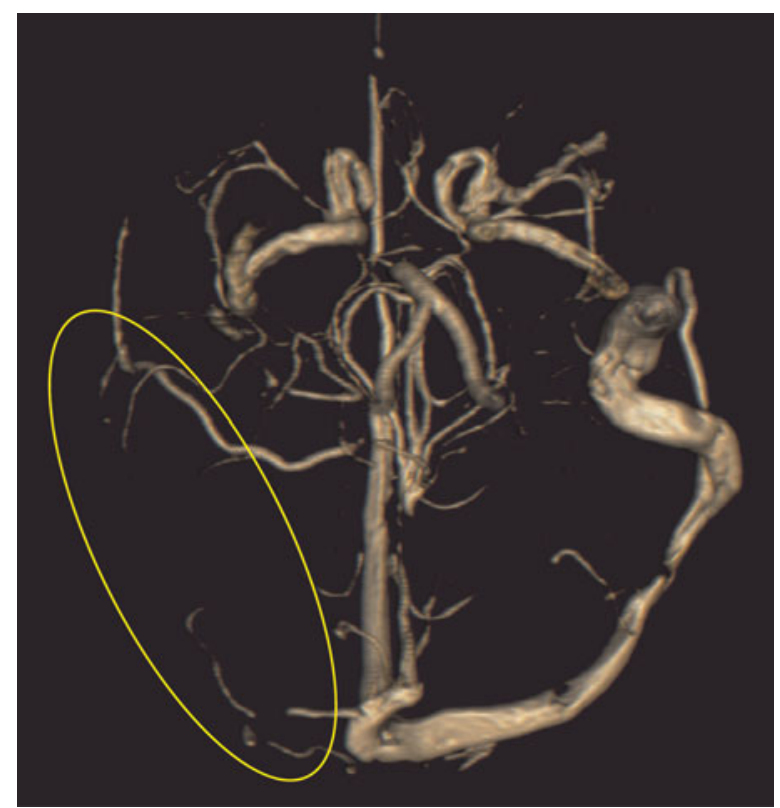

FIG. 3

Case three: reconstructed, three-dimensional computed tomography angiography showing venous thrombosis of the right transverse and sigmoid sinus (oval). 
A few days later, the patient's general condition deteriorated once again. A new CT scan showed a pneumocephalus due to erosion of the tegmen tympani resulting in a small temporal lobe abscess.

At further surgery, the abscess was drained and bony erosion was sealed in collaboration with the neurosurgeons. Intravenous antibiotics were given for 14 consecutive weeks. At the completion of treatment, there were some persistent neuropsychological sequelae (such as a degree of constructive apraxia, visual agnosia, handwriting alteration and memory problems), while other symptoms (such as time disorientation, speech organisation and general behaviour) were clearly ameliorated. There was no residual sensory or motor loss. Despite a transitory increase in venous glucose levels, the patient did not need permanent anti-diabetic treatment.

\section{Discussion}

Most non-ENT doctors consider malignant otitis externa to be a life-threatening form of otitis externa caused by $P$ aeruginosa in diabetic patients. However, the above three cases show that this condition may vary from the expected pattern of clinical presentation.

Pseudomonas aeruginosa is by far the commonest pathogen implicated in malignant otitis externa, being responsible for almost 98 per cent of all cases. ${ }^{4}$ Many other microbes can be implicated, including numerous bacteria (such as $S$ aureus, Staphylococcus epidermidis, Proteus mirabilis, Klebsiella oxytoca and Pseudomonas cepacia) and fungi (such as Candida ciferri and C parapsilosis, Malassezia sympodialis, Scedosporium apiosperumum, and Aspergillus fumigatus and $A$ niger). Cases of malignant otitis externa due to Aspergillus flavus, as in our first patient, are rare, and few cases have been reported. ${ }^{5,6}$

It is well known that diabetic patients are more prone to malignant otitis externa than healthy individuals. Nevertheless, 10 per cent of malignant otitis externa cases involve non-diabetic patients, as was the case with our second patient. ${ }^{7}$ Chronically ill and immunosuppressed patients are also at risk. ${ }^{8}$

Our third case illustrates the importance of early diagnosis, as the evolution of malignant otitis externa may not always be slow and gradual but sometimes rapid and fulminant. Our patient presented with a venous sinus thrombosis, a cerebral infarct and a cerebral abscess, just a few days after his initial symptoms of otitis externa. Other central nervous system complications, such as meningitis, have also been reported. ${ }^{9}$

Imaging studies are particularly useful in determining the extent of the inflammatory process. They lack specificity in differentiating malignant otitis externa from diseases with similar symptoms, such as nasopharyngeal and skull base tumours. ${ }^{8}$ In such cases, deep surgical biopsies are mandatory, and are now the main indication for surgery in patients with suspected malignant otitis externa. ${ }^{4}$ It is interesting that the initial extent of the inflammatory process as shown in the CT scan seems to correlate poorly with the outcome of the disease. ${ }^{10}$ Moreover, imaging studies such as CT and MRI should not be used to evaluate patients' responses to medical treatment. In our first case, a post-operative MRI taken after one month of antifungal treatment showed only slight improvement, although the patient had rapidly become asymptomatic. Interestingly, gallium 67 scanning, which was unfortunately not performed for any of our three patients, has been reported to return to normal after malignant otitis externa infection has cleared. Several studies have suggested it as a useful tool for deciding on treatment cessation and detecting recurrence. ${ }^{7}$
The presentation of vertigo in our second patient's case is particularly interesting. Persistent sensorineural hearing loss, third degree nystagmus and a positive head-thrust test suggested probable labyrinthitis. We have not found any similar cases reported. The otic capsule is considered to be an obstacle to the extension of disease to the inner ear, because the bony labyrinth demonstrates remarkable resistance to the osteomyelitic process. ${ }^{9,11}$

- Malignant otitis externa is traditionally perceived as a Pseudomonas aeruginosa external otitis resistant to local treatment in a diabetic patient

- This typical scenario is not always presented, as illustrated by this case series

- Diagnosis of malignant otitis externa is based on a combination of clinical findings, history, imaging, cultures and histology

- Skull base osteitis, seen in imaging studies, is an important diagnostic feature

It is widely accepted that clinical suspicion of malignant otitis externa should arise when otalgia persists in adequately treated patients with otitis externa. The diagnosis is rendered more probable by additional features from the history and physical examination, such as diabetes mellitus, immunodeficiency or cranial nerve palsy. As the therapeutic approach and prognosis for simple and malignant otitis externa differ drastically, the critical question to be answered concerns the criteria needed to diagnose malignant otitis externa. This necessity was identified by Cohen and Friedman in 1987 while trying to establish diagnostic criteria by reviewing 107 reported cases. ${ }^{12}$ A review of the literature reveals that there is no single pathognomonic feature of malignant otitis externa. The diagnosis is based on a combination of clinical findings, history, imaging, microbiology and histology. Identification of skull base osteitis in imaging studies seems to be an indispensable indicator. A bacterial or a fungal species should always be isolated. Histology's role is mainly in differentiating from malignant tumour.

\section{References}

1 Toulmouche MA. Observations on cerebral otorrhea: latest considerations [in French]. Gaz Med Paris 1838;6:422-6

2 Meltzer PE, Keleman G. Pyocutaneous osteomyelitis of the temporal bone, mandible and zygoma. Laryngoscope 1959;:169:1300-16

3 Chandler JR. Malignant external otitis. Laryngoscope 1968:78:1257-94

4 Rubin Grandis J, Branstetter BF 4th, Yu VL. The changing face of malignant (necrotizing) external otitis: clinical, radiological and anatomic considerations. Lancet Infect Dis 2004:4:34-9

5 Kountakis SE, Kemper JV Jr, Chang CY, DiMaio DJ, Stiernberg CM. Osteomyelitis of the base of the skull secondary to aspergillus. Am J Otolaryngol 1997;18:19-22

6 Mani R, Belcadhi M, Krifa N, Abdelkefi M, Ben Said M, Bouzouita K. Fungal necrotizing external otitis [in French]. Ann Otolaryngol Chir Cervicofac 2008;125:40-5

7 Carfrae MJ, Kesser BW. Malignant otitis externa. Otolaryngol Clin North Am 2008;41:537-49

8 Linstrom CJ, Lucente FE. Infections of the External Ear Bailey \& Johnson Head and Neck Surgery - Otolaryngology, 4th edn. Philadeplphia: Lippincott Williams \& Wilkins, 2006; 2:1994-7

9 Sreepada GS, Kwartler JA. Skull base osteomyelitis secondary to malignant otitis externa. Curr Opin Otolaryngol Head Neck Surg 2003;11:316-23

10 Sudhoff H, Rajagopal S, Mani N, Moumoulidis I, Axon PR, Moffat D. Usefulness of CT scans in malignant external 
otitis: effective tool for diagnosis, but of limited value in predicting outcome. Eur Arch Otorhinolaryngol 2008;265: $53-6$

11 Nadol JB Jr. Histophathology of Pseudomonas osteomyelitis of the temporal bone starting as malignant external otitis. Am J Otolaryngol 1980;1:359-71

12 Cohen D, Friedman P. The diagnostic criteria of malignant external otitis. J Laryngol Otol 1987;101:216-21

Address for correspondence:

Dr Zacharias Vourexakis

Department of Otolaryngology, Head and Neck Surgery,
University of Geneva School of Medicine,

Geneva University Hospitals,

Rue Gabrielle - Perret - Gentil 4,

1211 Geneva, Switzerland.

E-mail: zkvourexakis@yahoo.com

Dr Z Vourexakis takes responsibility for the integrity of the content of the paper.

Competing interests: None declared 